\title{
Recurrent bacterial meningitis occurring five years after closed head injury and caused by an intranasal post-traumatic meningo-encephalocele
}

\author{
Giovanni Giunta and Innocente Piazza
}

Department of Internal Medicine, Hospital of San Donà di Piave, Venice, Italy

Summary: A case of atypical presentation of a post-traumatic intranasal meningo-encephalocele is
described in a patient with a history of recurrent bacterial meningitis occurring 5 years after closed head
injury. The usefulness of the CT and MRI findings in diagnostic evaluation of this lesion is emphasized.

Introduction

An intranasal meningo-encephalocele or encephalocele consists of a discontinuity of the ethmoidal cribriform plate with protrusion of the meninges and brain tissue into the nose. It may be caused by congenital anomalies, ${ }^{1}$ by posttraumatic fracture of the ethmoidal cribriform plate, by rhinological operations, or by bone erosion due to raised intracranial pressure. ${ }^{2,3}$ Cerebrospinal fluid (CSF) rhinorrhoea and recurrent bacterial meningitis can complicate all types of meningo-encephalocele.

We report here a case of a patient with posttraumatic intranasal meningo-encephalocele, whose onset of recurrent bacterial meningitis occurred 5 years after closed head injury, and discuss the problems in the diagnostic evaluation of this lesion.

\section{Case report}

A 22 year old woman was admitted to the hospital with severe headache, fever, nausea and vomiting. Physical examination showed nuchal rigidity. A lumbar puncture revealed findings of purulent meningitis and a CSF culture showed a pneumococcal infection. After the infection was treated with ampicillin and chloramphenicol for 10 days, complete recovery without neurological consequences was obtained. The patient complained of 2 previous episodes of purulent meningitis due to

Correspondence: G. Giunta, Divisione Medica II, Ospedale Civile, Via Nazario Sauro 25, 30027 S. Donà di Piave, Venice, Italy

Accepted: 15 October 1990 pneumococcus that had occurred 3 and 7 months before. A complete investigation was performed to find out whether there was an immunological or anatomical defect causing these recurrent episodes of meningitis. Abnormalities of humoral and cellmediated immunity were not identified. Her delivery at birth had been normal, and there had been no rhinological operations.

She had, however, sustained head trauma with multiple skull fractures (frontal bone and maxillofacial area) 5 years before in a road accident. After the trauma she had a leakage of clear fluid (uncontaminated by blood) from her nose for only a few days. No external cranial deformities were present. Anosmia was not present. On admission to our hospital there was no CSF rhinorrhoea. Left rhinoscopy revealed the presence of a smooth solitary small nasal mass, which was soft, greyish and pulsating synchronously with the heart-beat, interpreted as a probable intranasal meningoencephalocele. A simple skull X-ray revealed no abnormalities. A computed tomographic (CT) scan of the skull performed without intravenous injection of contrast material, both in the axial and coronal incidences, showed a discontinuity of the ethmoidal cribriform plate at the base of the skull through which a small part of cerebral tissue with a diameter of about $1 \mathrm{~cm} \times 1 \mathrm{~cm}$ herniated into the left nasal cavity (Figure 1). Magnetic resonance imaging (MRI) of the brain, with coronal and sagittal views, confirmed that the intranasal mass was a meningo-encephalocele (Figure 2). The meningo-encephalocele was excised and the bone defect was repaired. The patient was well 5 months after surgery. 

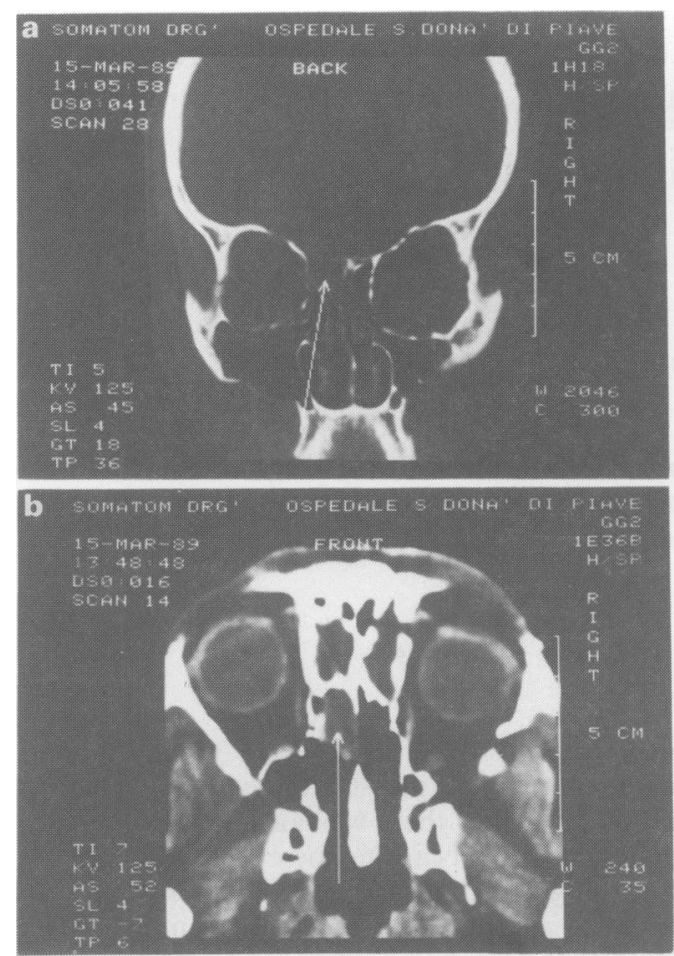

Figure 1 Computed tomographic scanning of the skull. The direct coronal (a) incidences clearly show the discontinuity in the cribriform plate and the brain tissue herniating into the left nasal cavity (meningoencephalocele). The axial (b) incidences show an oval mass in the left nasal cavity whose density is made up of cerebral tissue.

\section{Discussion}

The intranasal post-traumatic meningo-encephalocele is caused by a discontinuity of the ethmoidal cribriform plate as a consequence of a fracture at the base of the skull with herniation of the dura, the arachnoid and the brain into the fracture site. The cribriform plate is particularly fragile and vulnerable to fractures as a result of head trauma. CSF rhinorrhoea can develop at any time from hours to months ${ }^{4}$ after head injury, and recurrent bacterial meningitis usually develops within the first 3 months. ${ }^{5}$ Therefore, to our knowledge, a case of recurrent meningitis appearing 5 years after the original CSF rhinorrhoea appears to be unusual. The cessation of CSF leakage from the nose a few days after the head injury might have been due in our case to spontaneous closure of the fistula caused by adhesions or herniation of the brain into bone crevices. However, the quality of closure was inadequate and the risk of meningitis may remain even in the
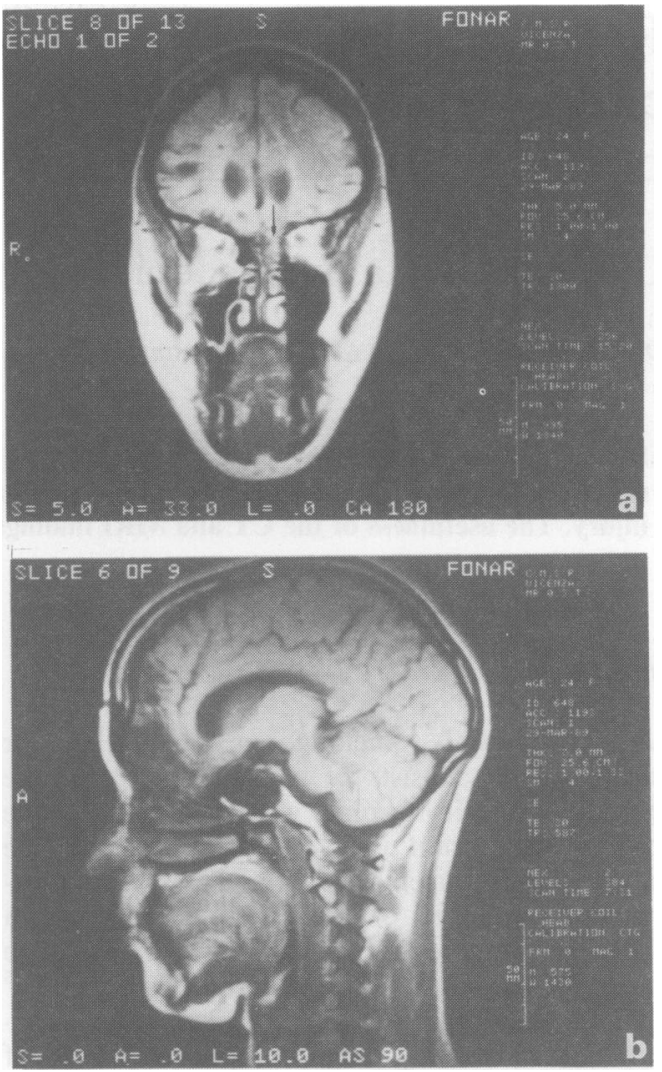

Figure 2 T1- and T2-weighted MRI of the brain in coronal (a) and sagittal (b) planes. Irregular atrophy of the anterior frontal lobe with dilated arachnoid space (aftermath of post-traumatic brain contusion). Acquired herniation of cerebral tissue, through a cribriform plate defect, into the left nasal cavity (meningo-encephalocele).

case of spontaneous closure of the fistula.

Both the congenital and post-traumatic types of intranasal meningo-encephalocele may be visible to inspection of the nasal fossae, appearing as a soft whitish mass; this must be distinguished from a polyp $^{6,7}$ and from other congenital intranasal masses - haemangiomas, dermoid cysts ${ }^{8}$ etc. because their removal may have undesirable consequences ${ }^{6,7}$ Differential diagnosis may be made by radiological studies. A simple skull X-ray does not give any clear clue. CT scans of the skull performed in the direct coronal incidences can locate exactly the defect in the ethmoidal cribriform plate, can demonstrate the herniation of the brain tissue into the nose, and can distinguish it from other intranasal masses that do not have intracranial extension. ${ }^{8}$ The axial CT scans can show up only a mass in a nasal cavity whose density is the same as brain tissue, but they give no significant images of 
the bone defect. MRI clearly shows the intranasal herniation of brain tissue and can distinguish it from the polyp and congenital masses. ${ }^{9}$ MRI is superior to CT due to its excellent soft tissue contrast resolution and does not require use of ionizing radiation. However, MRI does not demonstrate the bone defect as well as the CT scan, because no signal is generated from bone. ${ }^{9}$ The most significant views of MRI are coronal and sagittal.

The treatment of intranasal meningoencephalocele is surgical intervention. Prophylactic use of antibiotics is ineffective and should be abandoned. ${ }^{4,5}$ The surgical techniques have been described in detail ${ }^{5,10}$ and will not be described in this report. Surgical mortality is minimal in expert hands. $^{5}$ If surgical repair of the bone defect is successful the prognosis for the patient is excellent. $^{10}$

\section{References}

1. Blumenfeld, R. \& Skolnik, E.M. Intranasal encephaloceles. Arch Otolaryngol 1965, 82: 527-531.

2. Rovit, R.L., Schechter, M.M. \& Nelson, K. Spontaneous 'high pressure cerebrospinal rhinorrhea' due to lesions obstructing flow of cerebrospinal fluid. J Neurosurg 1969, 30: 406-412.

3. Von Essen, C. A large convexity meningioma followed by liquorrhoea from a nasal encephalocele. Acta Neurochir 1981, 59: 143-150.

4. Frazee, R.C., Mucha, P., Farnell, M.B. \& Ebersold, M.J. Meningitis after basilar skull fracture. Does antibiotic prophylaxis help? Postgrad Med 1988, 83: 267-268, 273-274.

5. Wilkins, R.H. \& Rengachary, S.S. (eds) Cerebrospinal fluid fistulas. In: Neurosurgery. McGraw-Hill, New York, 1985, 14: $277-291$

6. Jones, R.E., Bennington, J.L. \& Warner, N.E. Encephalocele masquerading as nasal polyp. JAMA 1962, 181: 640-642.
Recurrent bacterial meningitis, even without CSF rhinorrhoea, appearing several years after closed head injury, may be due to a concealed post-traumatic intranasal meningo-encephalocele. The case presented here demonstrates the usefulness of CT scanning (even if performed without contrast) and MRI in the pre-operative evaluation of nasal masses. Both CT and MRI clearly show the ethmoidal cribriform plate defect and the brain herniating into the nose. Therefore, in our case it has been possible to avoid more invasive investigations such as RISA-cisternography and CT-cisternography.

\section{Acknowledgement}

We thank Dr Giulio Vallis, consultant neuroradiologist, for his help in the radiological investigations.

7. Love, G.L. \& Rihel, P.A. Intranasal encephalocele masking as a nasal polyp in an adult patient. Arch Otolaryngol 1983, 109: 420-421.

8. Hemmy, D.C. Skeletal morphology of anterior encephaloceles defined throught the use of three dimensional reconstruction of computed tomography. Neurosciences 1985-1986; 12: 182.

9. Lusk, R.P. \& Lee, P.C. Magnetic resonance imaging of congenital midline nasal masses. Otolaryngol Head Neck Surg 1986, 95 (3 Pt 1): 303-306.

10. Spetzler, R.F. \& Wilson, C.B. Dural fistulae and their repair. In: Youmans, J.R. (ed.) Neurological Surgery, ed. 2, vol. 4. W.B. Saunders, Philadelphia, London, Toronto, etc, 1982, pp. 2209-2226. 